\title{
Radar Cross-Section Formulation of a Shell-Shaped Projectile Using Modified PO Analysis
}

\author{
Mohammad Asif Zaman and Md. Abdul Matin \\ Department of Electrical and Electronic Engineering, Bangladesh University of Engineering and Technology, Dhaka 1000, Bangladesh \\ Correspondence should be addressed to Mohammad Asif Zaman, asifzaman13@gmail.com
}

Received 30 April 2012; Revised 11 August 2012; Accepted 12 August 2012

Academic Editor: Azah Mohamed

Copyright ( $) 2012$ M. A. Zaman and Md. A. Matin. This is an open access article distributed under the Creative Commons Attribution License, which permits unrestricted use, distribution, and reproduction in any medium, provided the original work is properly cited.

A physical optics based method is presented for calculation of monostatic Radar Cross-Section (RCS) of a shell-shaped projectile. The projectile is modeled using differential geometry. The paper presents a detailed analysis procedure for RCS formulation using physical optics (PO) method. The shortcomings of the PO method in predicting accurate surface current density near the shadow boundaries are highlighted. A Fourier transform-based filtering method is proposed to remove the discontinuities in the approximated surface current density. The modified current density is used to formulate the scattered field and RCS. Numerical results are presented comparing the proposed method with conventional PO method. The results are also compared with published results of similar objects and found to be in good agreement.

\section{Introduction}

Prediction and measurement of Radar Cross-Section (RCS) have been a significant area of research for scientists and engineers for many years. The widespread uses of radar technology since the Second World War have demanded accurate and efficient prediction of fields scattered by radar targets. The knowledge of echo characteristics of radar targets is of great importance for the design of high-performance radars, as well as low visibility stealth targets $[1,2]$.

In radar technology, an antenna radiates electromagnetic (EM) energy. When an object is illuminated by the radar EM field, it reflects back some EM energy, which is received by an antenna. In monostatic radar system, the transmitting and reception of the EM energy are done by the same antenna or by multiple antennas located very close to each other $[1,3]$. In bistatic radar system, separate antennas are used for transmitting and receiving, and the antennas are usually far away from each other [1]. The radar field reflective nature of an object is specified in terms of RCS. The RCS is the area that a target would have to occupy to produce the amount of reflected power that is detected back at the radar $[2,4]$. The RCS of an object depends on the viewing angles, the size, geometry, and composition of the object, frequency and polarization of the radar signal, and so forth $[3,4]$. Stealth technology concentrates on reducing the RCS of airplanes and missiles to make them invisible to radars. Conversely, radar engineers are developing more sensitive radars that can detect low RCS targets. In both cases, accurate numerical simulation methods are essential for design purposes.

Numerical simulation of RCS of an object requires calculation of the scattered field from the object for a given incident field. Several numerical methods exist for scattered EM field calculations. Pure numerical methods such as Method of Moments (MoM), Finite-Difference TimeDomain (FDTD) method, Fast Multipole Method (FMM), and Transmission-Line Matrix (TLM) have been successfully used in predicting RCS of radar targets $[4,5]$. Conformal FDTD-based methods have also appeared in literature [6]. Recently, some variants of MoM have been developed for monostatic RCS formulation $[7,8]$ and other scattering related problems [9]. These methods do not depend on the geometry and can be used for any arbitrary shaped objects. However, these methods are computationally demanding, and the high simulation time for electrically large objects is not always acceptable for design and optimization problems. 
High-frequency asymptotic methods, such as Geometrical Optics (GO), Physical Optics (PO), Uniform Geometrical Theory of Diffraction (UTD), and Physical Theory of Diffraction (PTD), have also been used for RCS formulation [4]. These methods are based on local interaction of EM fields. Therefore, they are computationally less demanding than the pure numerical methods, and they require far less simulation time [10]. However, these methods are geometry dependent. For complex shaped objects, the scattered field formulation can be tedious, especially when using UTD method [11]. GO and PO methods do not suffer to the same extent as UTD for complex geometry cases. The GO method is the fastest among the high frequency techniques, but it is relatively less accurate [11]. The PO method gives much more accurate results compared to GO. It is a well-accepted method for formulating scattered field from electrically large objects $[12,13]$.

Because of its relatively high accuracy, the PO method has been widely used for RCS formulation $[14,15]$. The PO method is also computationally more efficient than pure numeric methods, making it relatively faster which makes it an essential tool for aerospace designers [16]. Several modified versions of PO have been developed to further increase the speed and accuracy of the PO method $[17,18]$. In this paper, a new modified PO method is used to formulate the monostatic RCS of a shell-shaped projectile.

This paper presents a detailed description and procedure of RCS calculation method of an object using PO method and modified PO method. The procedure includes the geometrical modeling of the shell-shaped radar object, approximation of the induced surface current on the object, and formulation of the $\mathrm{PO}$ radiation integral in parametric space. Although the paper concentrates on RCS formulation of a specific shell-shaped radar target, the analysis procedure is general and can be applied to objects of any geometry. In spite of the presence of many research and review articles [4], a complete description of PO method-based procedure for RCS calculation is rare in literature. In addition to the complete calculation procedure, this paper also presents a new modified PO method. The PO method approximates the induced surface current on the radar target and uses this current to calculate the scattered field [11, 19]. This approximation leads to discontinuous surface current across the target surface near the shadow boundaries [19]. In the proposed method, a better approximate of the induced surface currents is used to remove the unnatural discontinuities and improve the accuracy in calculation of the scattered field. The proposed method incorporates filtering inspired Fourier transform-based methods to remove the current discontinuity.

The paper is arranged as follows: in Section 2, the geometrical modeling of the shell-shaped projectile is described. Differential geometry-based definition of the surfaces and normals is derived here. Section 3 contains approximation of the induced surface current on object and formulation of the scattered field and RCS. Numerical results are presented in Section 4. Finally, concluding remarks are given in Section 5.

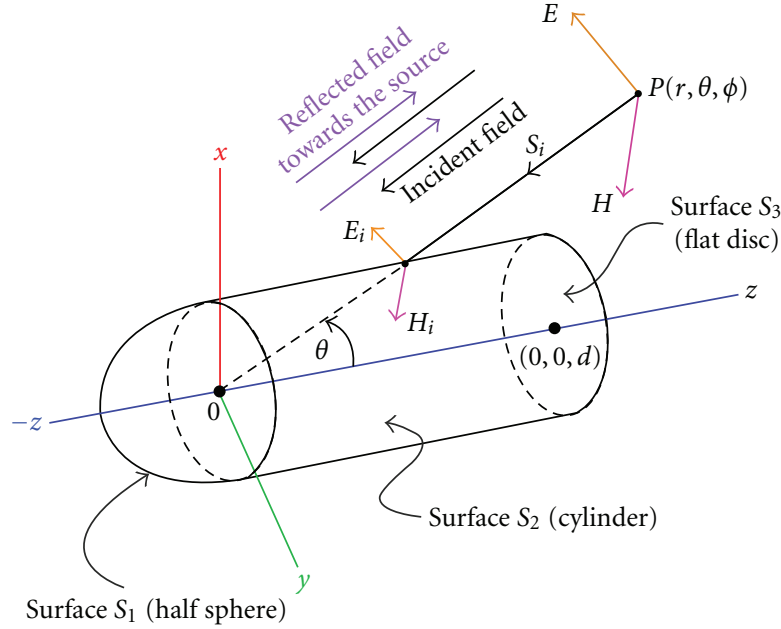

Figure 1: Three dimensional geometry of the shell-shaped projectile, and the coordinate system along with incident field vectors.

\section{Geometrical Modeling}

The first step in simulating the RCS of an object is to accurately model the surface of the object. This paper concentrates on the RCS of a shell-shaped object. A shellshaped object is selected as most projectiles represent this basic shape. A schematic diagram of the object and the threedimensional coordinate system is shown in Figure 1. The object can be modeled by three different canonical surfaces: a half sphere (surface $S_{1}$ ), a cylinder (surface $S_{2}$ ), and a flat disc (surface $S_{3}$ ). Most objects can be similarly modeled by a few common canonical shapes. For this reason, scattering from common shapes such as flat plates, cones, and cylinders has received attention in literature since the 1960s [20,21].

The origin of the coordinate system is taken at the center of the half sphere, and $z$ axis is taken as the axis of the cylinder. The three surfaces can be expressed by the following equations:

$$
\begin{gathered}
S_{1}: x^{2}+y^{2}+z^{2}=b^{2}, \quad-b \leq z \leq 0, \\
S_{2}: x^{2}+y^{2}=b^{2}, \quad 0 \leq z \leq d, \\
S_{3}: x^{2}+y^{2}=\rho^{2}, \quad 0 \leq \rho \leq b, z=d .
\end{gathered}
$$

Here, $b=$ radius of the half-sphere $=$ radius of the cylinder, and $d=$ length of the cylinder. For scattering problems and RCS formulation, it is often more convenient to express the surfaces in differential geometry format rather than coordinate geometry format [11]. To express the surfaces in differential geometry format, the following parameters are used:

$$
\begin{aligned}
& S_{1}: x=\rho_{s} \cos \phi_{s}, \quad y=\rho_{s} \sin \phi_{s}, \quad z=-\sqrt{b^{2}-\rho_{s}^{2}}, \\
& 0 \leq \rho_{s} \leq b, 0 \leq \phi_{s} \leq 2 \pi, \\
& S_{2}: x=b \cos \phi_{s}, \quad y=b \sin \phi_{s}, \quad z=\rho_{s}, \\
& 0 \leq \rho_{s} \leq d, 0 \leq \phi_{s} \leq 2 \pi,
\end{aligned}
$$




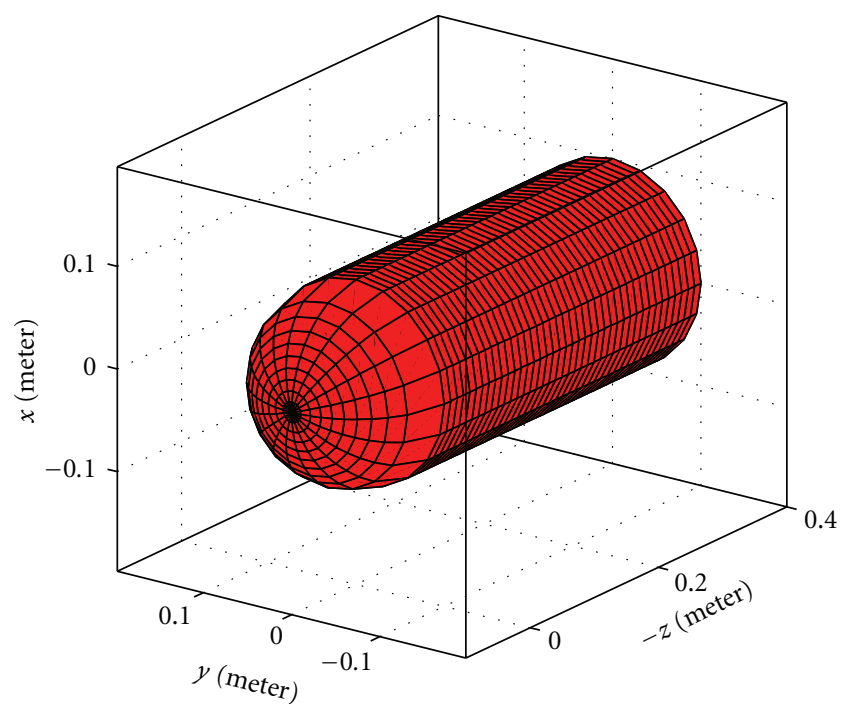

Figure 2: Computer-generated three-dimensional model of the shell-shaped projectile.

$$
\begin{aligned}
& S_{3}: x=\rho_{s} \cos \phi_{s}, \quad y=\rho_{s} \sin \phi_{s}, \quad z=d, \\
& 0 \leq \rho_{s} \leq b, 0 \leq \phi_{s} \leq 2 \pi .
\end{aligned}
$$

Here, $\rho_{s}$ and $\phi_{s}$ are parameters. Note that the $\rho_{s}$ and $\phi_{s}$ for $S_{1}$ are not the same as the $\rho_{s}$ and $\phi_{s}$ for $S_{2}$ or $S_{3}$. For each surface the parameters are different and unrelated. Same parameter names are used for simplicity only. Using these parameters, the differential geometric expressions of the surfaces are [22]

$$
\begin{gathered}
S_{1}: \mathbf{r}=p_{s} \cos \phi_{s} \hat{\mathbf{x}}+p_{s} \sin \phi_{s} \hat{\mathbf{y}}-\sqrt{b^{2}-\rho_{s}^{2}} \hat{\mathbf{z}}, \\
S_{2}: \mathbf{r}=b \cos \phi_{s} \hat{\mathbf{x}}+b \sin \phi_{s} \hat{\mathbf{y}}+\rho_{s} \hat{\mathbf{z}}, \\
S_{3}: \mathbf{r}=p_{s} \cos \phi_{s} \hat{\mathbf{x}}+p_{s} \sin \phi_{s} \hat{\mathbf{y}}+d \hat{\mathbf{z}} .
\end{gathered}
$$

The limits of the parameters are given in (2). Using these equations, it is possible to construct a three-dimensional model of the shell-shaped projectile using computer coding. The computer generated model is shown in Figure 2. For computation, $b=10 \mathrm{~cm}$ and $d=40 \mathrm{~cm}$ are used.

Once the surfaces of the object are defined, it is necessary to define normal vectors on each point of the surface. These normal vectors are necessary for GO-, UTD-, or PO-based scattering formulations [19]. The normal vectors, $\hat{\mathbf{n}}$, can be calculated from the differential geometric expression of the surfaces using the following equation $[11,22]$ :

$$
\hat{\mathbf{n}}= \pm \frac{\left(\partial \mathbf{r} / \partial \rho_{s}\right) \times\left(\partial \mathbf{r} / \partial \phi_{s}\right)}{\left|\left(\partial \mathbf{r} / \partial \rho_{s}\right) \times\left(\partial \mathbf{r} / \partial \phi_{s}\right)\right|}
$$

The sign of the normal vectors is selected so that they always point away from the surface. Using (4), the normal vectors on the three surfaces of the object are calculated to be

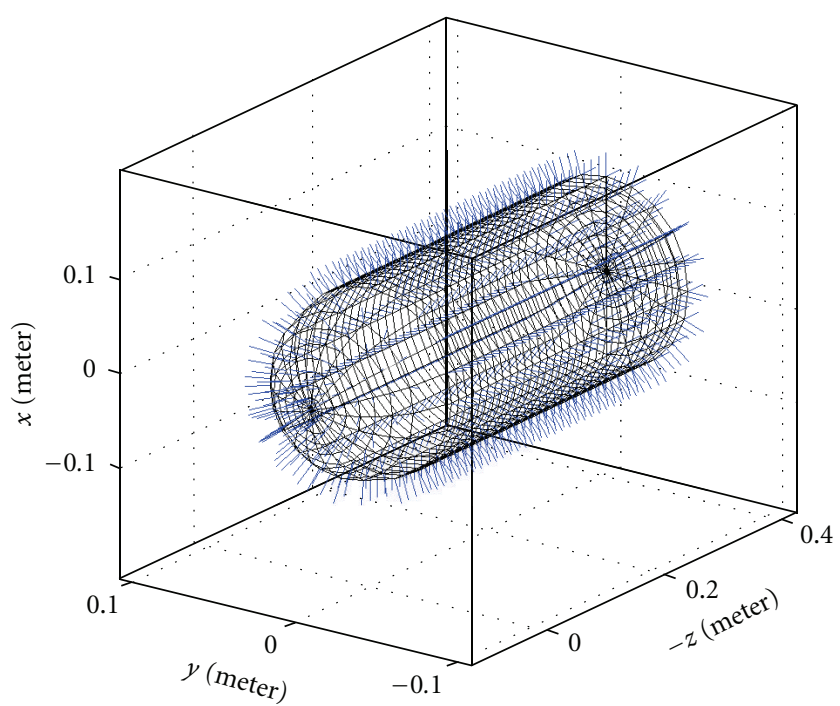

FIgURE 3: Normal vectors (blue lines) on the object surfaces.

$$
\begin{gathered}
S_{1}: \hat{\mathbf{n}}=\frac{\rho_{s}}{b} \cos \phi_{s} \hat{\mathbf{x}}+\frac{\rho_{s}}{b} \sin \phi_{s} \hat{\mathbf{y}}-\frac{\sqrt{b^{2}-\rho_{s}^{2}}}{b} \hat{\mathbf{z}}, \\
S_{2}: \hat{\mathbf{n}}=\cos \phi_{s} \hat{\mathbf{x}}+\sin \phi_{s} \hat{\mathbf{y}} \\
S_{3}: \hat{\mathbf{n}}=\hat{\mathbf{z}} .
\end{gathered}
$$

Using (5), the normal vectors are plotted over the wire frame of the object using computer coding. The results are shown in Figure 3. From visual inspection it is verified that the normals are perpendicular to the surface and points away from it. This verifies the geometrical modeling performed in this section.

\section{RCS Formulation Using Modified PO Method}

To formulate the RCS of the object, the incident field must be defined first. As the radar and the target are usually very far away from each other, the incident field can be modeled as a plane wave, implying that the direction of the wave, the direction of the electric field, and the direction of the magnetic field are perpendicular to each other. With respect to a coordinate system defined at the source point of the wave, if the wave travels at $z$ direction, and the electric field is assumed to be polarized along $x$ direction, then the magnetic field will be polarized along $y$ direction. However, in this case, the coordinate system is defined with respect to the object. So, coordinate transformation must be performed to find the expression of the incident field with respect to the defined coordinate system $[23,24]$. For a wave that is incident on the angle $(\theta, \phi)$, an $x$ direction polarized electric field converts to a $\theta$ polarized wave [24]. So, the incident electric field can be expressed as $[11,24]$

$$
\mathbf{E}_{\mathbf{i}}=\left|\mathbf{E}_{\mathbf{i}}\right| \hat{\theta}=\cos \theta \cos \phi \hat{\mathbf{x}}+\cos \theta \sin \phi \hat{\mathbf{y}}+\sin \theta \hat{\mathbf{z}} .
$$

Here, the amplitude of the incident electric field is assumed to be 1 . The direction of the incident ray is given by

$$
\widehat{\mathbf{s}}_{\mathbf{i}}=\sin \theta \cos \phi \hat{\mathbf{x}}+\sin \theta \sin \phi \hat{\mathbf{y}}-\cos \theta \hat{\mathbf{z}} \text {. }
$$


The incident ray direction, along with electric and magnetic field polarizations, is shown in Figure 1.

For monostatic RCS calculation, the reflected ray is

$$
\hat{\mathbf{s}}_{\mathbf{r}}=-\hat{\mathbf{s}}_{\mathbf{i}}
$$

PO method uses approximate expression of the surface current density induced on the surface of the object due to the incident field to find the scattered field. The surface current density depends on the incident magnetic field. The incident magnetic field is given by $[11,19]$

$$
\mathbf{H}_{\mathbf{i}}=\frac{1}{\eta}\left(\widehat{\mathbf{s}}_{\mathbf{i}} \times \mathbf{E}_{\mathbf{i}}\right) .
$$

Here, $\eta=120 \pi=$ intrinsic impedance of air. Equation (9) is valid only for plane wave incidence. As the object can be considered to be very far away from the source for most radar applications, this assumption is justified. The PO approximates the surface current density, $\mathbf{J}_{\mathbf{P O}}$, as [18]

$$
\mathbf{J}_{\mathbf{P O}}= \begin{cases}2 \hat{\mathbf{n}} \times \mathbf{H}_{\mathbf{i}}, & \text { for illuminated surface, } \\ 0, & \text { otherwise. }\end{cases}
$$

Equation (10) holds for a perfectly conduction object. For calculating RCS of a metallic object, this approximation is justified. Using (7), (9) along with (5) in (10), it is possible to calculate $\mathbf{J}_{\mathbf{P O}}$. However, it is necessary to identify which part of the surface is illuminated by the incident field and which part is not for calculation of (10). This can be accomplished by noticing the angle between $\widehat{\boldsymbol{s}}_{\mathbf{i}}$ and $\hat{\mathbf{n}}$. When the vectors are perpendicular to each other, the incident field is tangent on the surface $[22,23]$. These surface points indicate the shadow boundary, beyond which the surface points will not be illuminated. So, surface points on which the angle between the normal vector and incident ray vector is greater than $90^{\circ}$ are in the shadowed region. This statement can be mathematically expressed as

$$
\begin{aligned}
& \hat{\mathbf{n}} \cdot \widehat{\mathbf{s}}_{\mathbf{i}} \leq 0 \text { for illuminated region, } \\
& \hat{\mathbf{n}} \cdot \widehat{\mathbf{s}}_{\mathbf{i}}>0 \text { for shadowed region. }
\end{aligned}
$$

Thus using (10) and (11), the PO approximate of the surface current density can be formulated on $S_{1}, S_{2}$, and $S_{3}$ surfaces. It should be mentioned that (11) is true only for objects that only have convex surfaces. If one region of the object creates shadow for another region, then (11) cannot be used to identify the illuminated region and shadowed region. For the relatively simple geometry presented in this paper, (11) is sufficient for calculating the shadow boundaries.

From $\mathbf{J}_{\mathbf{P O}}$, the magnetic vector potential, $\mathbf{A}$ can be calculated using the radiation integral $[12,19]$ :

$$
\begin{aligned}
\mathbf{A}= & \int \mathbf{J}_{\mathbf{P O}} \frac{e^{-j k R}}{R} d S=\int \mathbf{J}_{\mathbf{P O}} \frac{e^{-j k R}}{R} d S_{1}+\int \mathbf{J}_{\mathbf{P O}} \frac{e^{-j k R}}{R} d S_{2} \\
& +\int \mathbf{J}_{\mathbf{P O}} \frac{e^{-j k R}}{R} d S_{3} .
\end{aligned}
$$

Here, $R$ is the distance from the object to the receiver. As monostatic radar cross-section is considered, the source and the receiver are located at the same position. The position of the source point is expressed in polar coordinates as $P(r, \theta, \phi)$ with $r=1000$ meter. $R$ can easily be calculated from the coordinates of the source and the object. The surface integrals can be performed over parametric space. The differential surface element $d S$ can be expressed in terms of the parameters as $[22,23]$

$$
d S=\left|\frac{\partial \mathbf{r}}{\partial \rho_{s}} \times \frac{\partial \mathbf{r}}{\partial \phi_{s}}\right| d \rho_{s} d \phi_{s}
$$

Evaluating (13) for the three surfaces and using it in (12):

$$
\begin{aligned}
\mathbf{A}= & \int_{\phi_{s}=0}^{2 \pi} \int_{\rho_{s}=0}^{b} \mathbf{J}_{\mathbf{P O}}\left(\rho_{s}, \phi_{s}\right) \frac{e^{-j k R}}{R} \frac{\rho_{s} b}{\sqrt{b^{2}-\rho_{s}^{2}}} d \rho_{s} d \phi_{s} \\
& +\int_{\phi_{s}=0}^{2 \pi} \int_{\rho_{s}=0}^{d} \mathbf{J}_{\mathbf{P O}}\left(\rho_{s}, \phi_{s}\right) \frac{e^{-j k R}}{R} b d \rho_{s} d \phi_{s} \\
& +\int_{\phi_{s}=0}^{2 \pi} \int_{\rho_{s}=0}^{b} \mathbf{J}_{\mathbf{P O}}\left(\rho_{s}, \phi_{s}\right) \frac{e^{-j k R}}{R} \rho_{s} d \rho_{s} d \phi_{s} .
\end{aligned}
$$

The scattered field, $\mathbf{E}_{\boldsymbol{s}}$, can be formulated from $\mathbf{A}$ as [12]

$$
\mathrm{E}_{\mathrm{s}}=-j \frac{60 \pi}{\lambda} \mathbf{A} \text {. }
$$

Here, $\lambda=$ wavelength of the incident field. The RCS, $\sigma$ of the object is calculated from the scattered field using (6) and (15) $[1,4]$ :

$$
\sigma=\lim _{R \rightarrow \infty} 4 \pi R^{2} \frac{\left|\mathbf{E}_{\mathbf{s}}\right|^{2}}{\left|\mathbf{E}_{\mathbf{i}}\right|^{2}} .
$$

The method described so far is the conventional PO method. Although sufficiently accurate, one of the weak points of this method is the approximation of the surface current density, $\mathbf{J}_{\mathbf{P O}}$. From (10), it can be seen that $\mathbf{J}_{\mathbf{P O}}$ abruptly changes to zero in the boundary between illuminated and shadow region. Practical surface current densities do not have this discontinuous nature. This discontinuity arises because PO method does not take into account the creeping waves which exist in the boundary between shadowed region and illuminated region. These waves gradually decrease with distance from the shadow boundaries, and the resulting surface current density decreases gradually.

So, to increase the accuracy of the PO method, the discontinuity $\mathbf{J}_{\mathbf{P O}}$ in must be removed. In this paper, a filteringbased approach is proposed to remove the discontinuity in current distribution. The spatial variation in the current distribution over the surface of the object can be compared with temporal variation of an analog signal in time domain. In Fourier expansion of time signals, the time signal is imagined as superimposition of many sinusoidal signals, termed Fourier spectral components. A sharply varying time signal has high Fourier spectral components. A discontinuous signal has large high Fourier spectral components. Using this analogy, the spatial variation of the surface current in scattering problems can be imagined as superimposition of many surface current components with sinusoidal variation. 
These components can be isolated using Fourier transformation. It can be imagined that the rapidly varying high components of the surface current are responsible for the sharp discontinuous spatial distribution. The high spectral components add in opposite phase with the other terms in the shadow region to create destructive interference. In absence of these high spectral components, the other components will not completely cancel each other, and therefore there will be an oscillating distribution of surface current in the shadow region. These oscillating distributions can be compared to the creeping waves. Thus filtering out high spectral components should create a smooth distribution of surface current densities which may accurately model the actual current distribution with a higher degree of accuracy.

To perform filtering operations to make $\mathbf{J}_{\mathbf{P O}}$ continuous, Fourier transform is performed in the parametric space. This produces Fourier domain representation of the PO surface current, JFPO. This can be obtained using the following relation [24]:

$$
\begin{aligned}
\mathbf{J}_{\mathrm{FPO}}\left(\rho_{s}, \zeta\right) & =F_{\phi_{s}}\left[\mathbf{J}_{\mathbf{P O}}\left(\rho_{s}, \phi_{s}\right)\right] \\
& =\frac{1}{\sqrt{2 \pi}} \int_{-\infty}^{\infty} \mathbf{J}_{\mathbf{P O}}\left(\rho_{s}, \phi_{s}\right) e^{-j \zeta \phi_{s}} d \phi_{s} .
\end{aligned}
$$

Here, Fourier transform is performed with respect to $\phi_{s}$, and $\zeta$ is another parameter corresponding to the frequency term in conventional Fourier transform of time series data. The complete information of $\mathbf{J}_{\mathbf{P O}}$ is implicit within $\mathbf{J}_{\mathbf{P P O}}$. The discontinuity in $\mathbf{J}_{\mathbf{P O}}$ with respect to $\phi_{s}$ will result in high $\mathbf{J}_{\mathrm{FPO}}$ values for large $\zeta$. This is analogous to high frequency terms in discontinuous time series data [25]. As these high $\zeta$ components contribute to the discontinuity, removing them should result in a smoother surface current density. How many high $\zeta$ value components need to be removed to produce an accurate surface current density cannot be analytically calculated due to the complex nature of the mathematics. Observing the discontinuity in the PO current in many canonical problems found in literature [11] and testing the filter for different parameters, it is found through trial and error that removing $70 \%$ of high $\zeta$ value components results in a relatively accurate surface current distribution. A modified current density, $\mathbf{J}_{\mathbf{S P O}}$, is constructed in $\zeta$ space by removing $70 \%$ of high $\zeta$ value components:

$$
\mathbf{J}_{\mathrm{SPO}}\left(\rho_{s}, \zeta\right)= \begin{cases}0, & \zeta \geq 0.7 \zeta_{\max } \\ \mathbf{J}_{\mathrm{FPO}}\left(\rho_{s}, \zeta\right), & \text { otherwise }\end{cases}
$$

Now, the modified smoother current density, $\mathbf{J}_{\mathbf{M P O}}$, in $\rho_{s}-\phi_{s}$ space can be obtained by using inverse Fourier transform [24]:

$$
\begin{aligned}
\mathbf{J}_{\mathbf{M P O}}\left(\rho_{s}, \phi_{s}\right) & =F_{\zeta}^{-1}\left[\mathbf{J}_{\mathbf{S P O}}\left(\rho_{s}, \zeta\right)\right] \\
& =\frac{1}{\sqrt{2 \pi}} \int_{-\infty}^{\infty} \mathbf{J}_{\mathbf{P O}}\left(\rho_{s}, \zeta\right) e^{j \zeta \phi_{s}} d \zeta .
\end{aligned}
$$

Equation (19) is applicable for canonical geometries and surface of revolution. Now, the discontinuity in $\mathbf{J}_{\mathbf{P O}}$ with respect to $\phi_{s}$ should no longer be present in $\mathbf{J}_{\mathrm{MPO}}$. However,

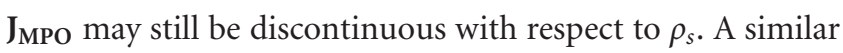
analysis can be performed to remove this discontinuity by taking Fourier transform of $\mathbf{J}_{\text {MPO }}$ with respect to $\rho_{s}$, eliminating high components and then taking inverse Fourier transform. Then a smoothed surface current density, $\mathbf{J}_{\mathbf{M}}$, can be obtained which is continuous across the surface of the object.

It is noted that that as some components of the current densities are filtered out, the energy of the conventional PO current may not be equal to the energy of the modified PO current. This may not be consistent with conservation of energy. To rectify this problem, the filtered current densities must be multiplied by a constant scalar so that the resulting current densities have the same energy as the PO current. The value of the constant can easily be calculated by calculating the energy of the conventional PO current and the unscaled modified PO current. This scaling ensures that no changes have occurred in total energy of the surface currents.

Due to harmonic oscillating nature of Fourier transform, $\mathbf{J}_{\mathbf{M}}$ has oscillating characteristics near the shadow boundary which accurately represents diffraction patterns. Using $\mathbf{J}_{\mathbf{M}}$ instead of $\mathbf{J}_{\mathbf{P O}}$ in (12)-(16) will result in a more accurate estimate of RCS.

\section{Numerical Results}

For numerical analysis, the frequency of the incident field is taken to be $10 \mathrm{GHz}$. The radius of the sphere and cylinder, $b=10 \mathrm{~cm}$, and the length of the cylinder, $d=40 \mathrm{~cm}$, are assumed. To perform numerical analysis, the surface of the object must be divided into discrete points. Discrete values of the parameters $\rho_{s}$ and $\phi_{s}$ are taken to create discrete points on the surface. Large number of points increase accuracy but also take considerable simulation time. Here, 22 values $\rho_{s}$ per wavelength are considered, and 204 values of $\phi_{s}$ per $\rho_{s}$ are considered. These values fall in the range of typical selected values for numerical analysis using PO type method [19]. All numerical results are obtained using computer coding.

Figures 4 and 5 show the surface current density for two different incident field orientations. These current densities are obtained using conventional PO methods using (10). The discontinuity in the current densities can easily be spotted in both figures. All the current densities are plotted after normalization process. The normalization is performed by dividing the current densities by the maximum value of the current density. Due to this division, the normalized current density is unitless. The resulting normalized current densities are expressed in decibels by taking logarithm.

Using the proposed modified PO method, the surface current densities are smoothed. Fourier transform is used with respect to both parameters. The resulting smoothed current densities are shown in Figures 6 and 7. The difference in current densities can easily be observed. Comparing Figure 4 with Figure 6 and Figure 5 with Figure 7, it can be seen that the current densities decrease gradually when the proposed method is used whereas the conventional PO method creates sharp discontinuities. The oscillating pattern of the currents near the shadow boundary is created when the proposed method is used. These accurately describe realistic diffracted fields [11]. 


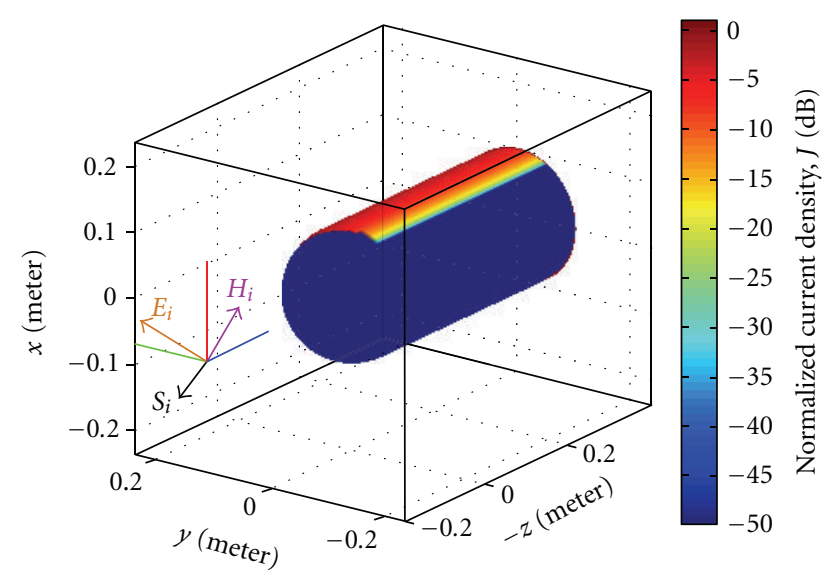

FIgURE 4: Surface current density using PO method for an incident field $\theta=20^{\circ}, \phi=60^{\circ}$.

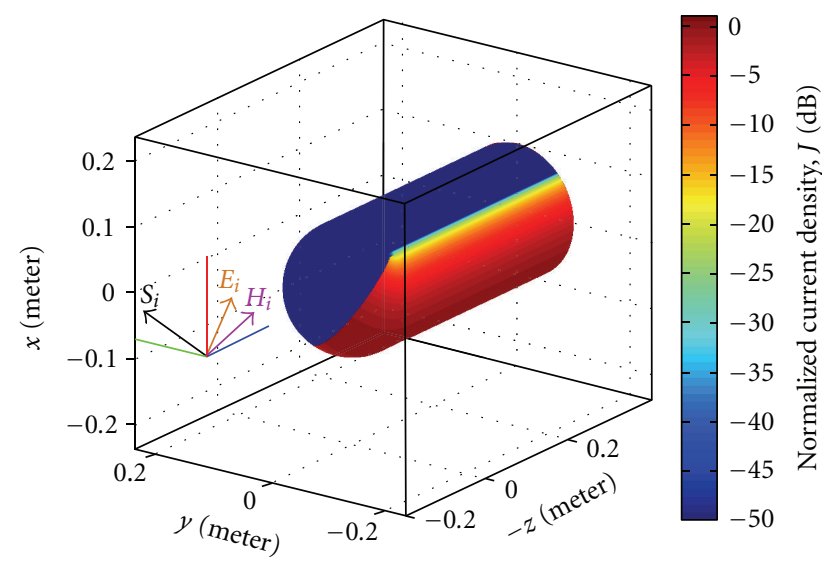

FIGURE 5: Surface current density using PO method for an incident field $\theta=60^{\circ}, \phi=45^{\circ}$.

To verify accuracy of the surface current densities approximated in the proposed filtering method, a simplified canonical problem of scattering from an infinite cylinder is considered. Considering an infinitely long cylinder with axis parallel to the $z$ axis is illuminated by a plane wave. The orientation of the magnetic field of the incident wave is considered along the $z$ axis, and the orientation of the electric field is assumed to be along the $x$ axis. The wave propagates along the $y$ axis. The PO current given by (10) is constant throughout the illuminated region of the cylinder. There will be no variation of the surface current along the $z$ axis. The plane wave will illuminate only half the surface of the cylinder. If an angle, $\chi$, is defined along the $x y$ plane as the angle from the $y$ axis, then for a plane wave propagating along the $y$ axis, the illuminated region spans from $\chi=-90^{\circ}$ to $90^{\circ}$. The PO current abruptly falls to zero outside this angle. The proposed filtering method adjusts this discontinuity and makes the current smooth. The actual angular distribution of the current density can be formulated using a Method of Moments (MoM), which is a well-known benchmark method [19]. The current distribution obtained

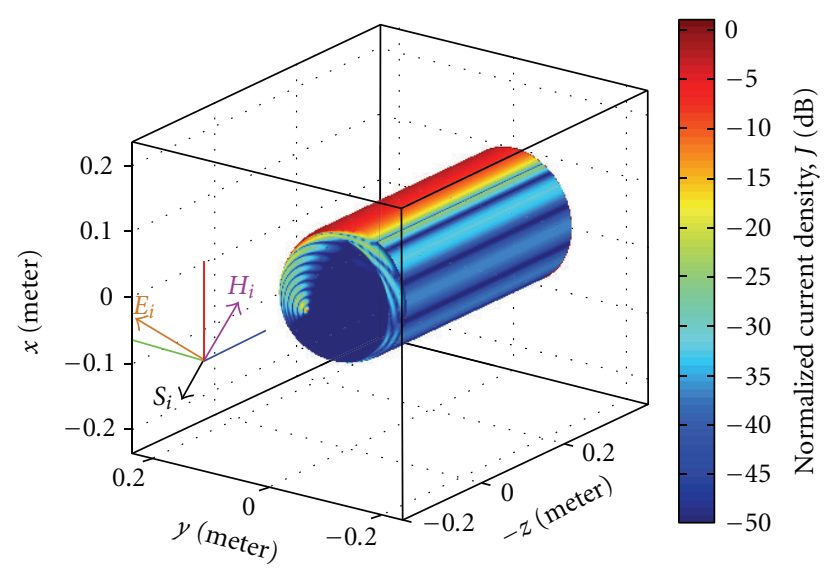

Figure 6: Surface current density using modified PO method for an incident field $\theta=20^{\circ}, \phi=60^{\circ}$.

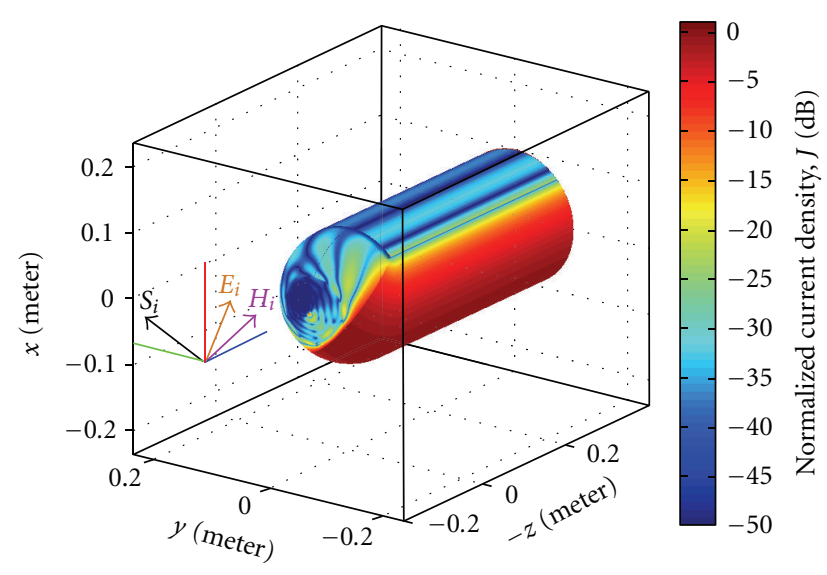

FIGURE 7: Surface current density using modified PO method for an incident field $\theta=60^{\circ}, \phi=45^{\circ}$.

from the PO method, the proposed modified PO method, and MoM are shown in Figure 8. It can be clearly observed that the proposed method produces a current distribution which resembles the results obtained from MoM much closely compared to the PO method. This higher degree of accuracy in estimating the surface current ensures that the proposed method will generate more accurate RCS results compared to the conventional PO method.

The RCS of the shell-shaped object is formulated using both conventional PO and the modified PO method. The RCS at $\phi=0^{\circ}$ plane as a function of zenith angle, $\theta$, is shown in Figure 9.

RCS is expressed in $\mathrm{dB}$ with respect to $1 \mathrm{~m}^{2}$ area. This unit is represented as $\mathrm{dB} \mathrm{m}^{2}$ or $\mathrm{dB} \mathrm{sm}[1,4]$. As the object is circularly symmetric around the $z$ axis, the scattered field and RCS are independent of the azimuth angle, $\phi[11,26]$. It is seen that both methods give similar results in most angular regions. The variation in result comes for angular regions where the spherical surface is illuminated. This is expected as the spherical surfaces are affected by the diffracted rays and creeping waves more than the other shaped surfaces 


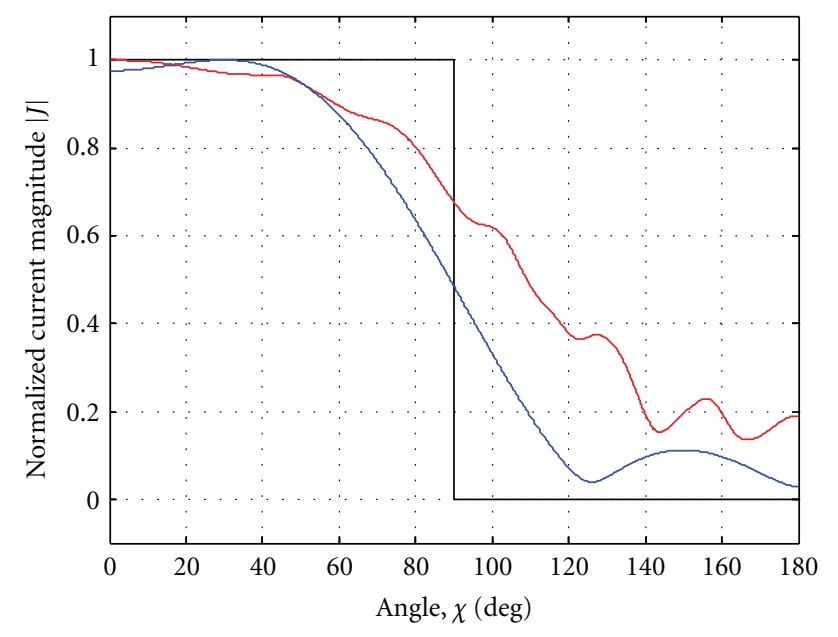

— PO approximation
- MoM solution
- Proposed method

FIGURE 8: Surface current densities on an infinite cylinder surface at $f=3 \mathrm{GHz}$.

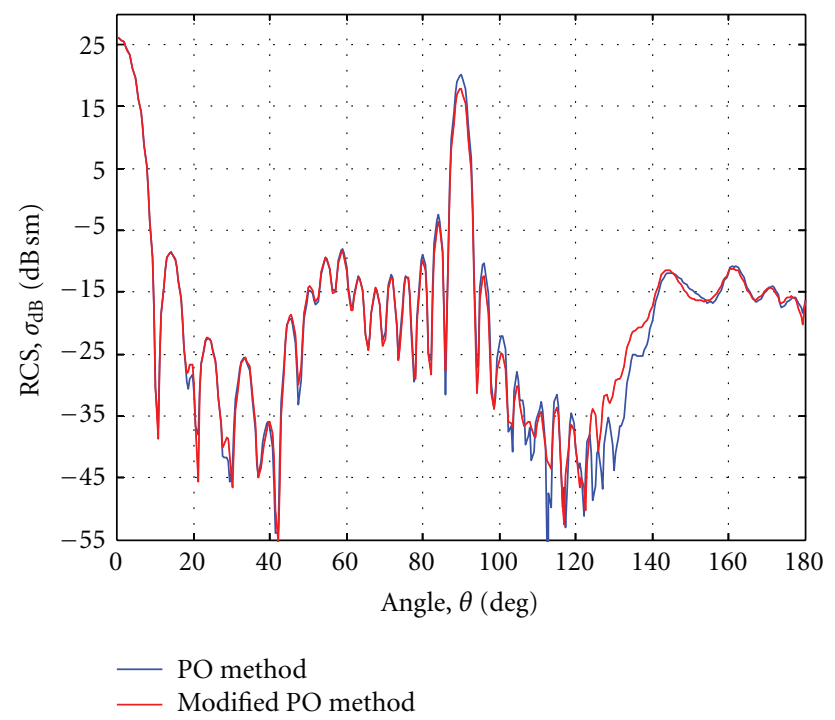

FIGURE 9: Monostatic RCS of the shell-shaped projectile at $10 \mathrm{GHz}$ as a function of $\theta$.

[11]. Due to the better approximate of the surface current, the results obtained from the proposed method are more accurate. It is noted that at $\theta=180^{\circ}$ only the half-sphere surface is illuminated. The RCS at this angle is around $-15 \mathrm{~dB} \mathrm{sm}$. The projected area of the half sphere $=\pi b^{2}=$ $0.0314 \mathrm{~m}^{2}=-15 \mathrm{~dB} \mathrm{sm}$, which is equal to the RCS. This is expected for a spherical shaped object. This consistency verifies the numerical analysis.

The RCS of the projectile at $5.5 \mathrm{GHz}$ as a function $\theta$ is shown in Figure 10. The results from the proposed method and MoM are shown in the same plot for comparison. It can be observed that the results obtained from the proposed

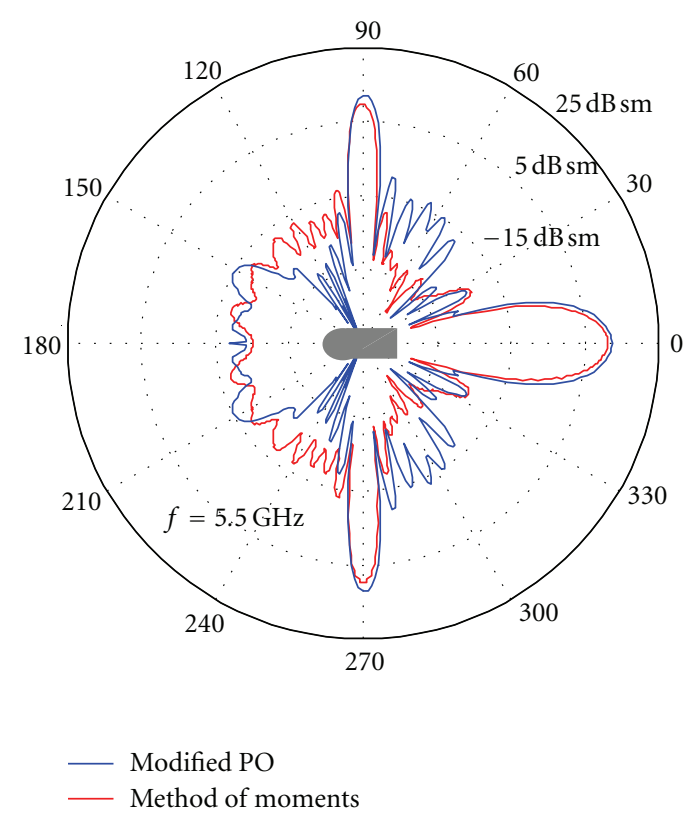

Figure 10: Monostatic RCS of the shell-shaped projectile at $5.5 \mathrm{GHz}$ as a function of $\theta$.

method matches with the results obtained from MoM closely for most values of $\theta$.

For the angles where RCS is high, the results of both methods are almost identical. The deviation arises for angles where RCS value is low. The higher degree of accuracy obtained from MoM comes at a cost of complex computation and extensive simulation time. The errors produced by the proposed method are relatively small and acceptable for many applications. In most cases the accuracy from PO method is sufficient, and the proposed method is expected to be more accurate than the PO method.

The RCS of the shell-shaped projectile for frequencies $3 \mathrm{GHz}, 6 \mathrm{GHz}, 10 \mathrm{GHz}$, and $14 \mathrm{GHz}$ as a function $\theta$ is shown in Figure 11. The obtained RCS pattern is similar to the RCS patterns of objects of similar shape described in $[4,16]$.

The computer simulation was performed on an Intel Core i5-2430M 2.4 GHz CPU with 2.94 GB usable RAM. The simulation time of conventional PO and modified PO is compared in Table 1.

From Table 1, it can be seen that for lower frequency, the simulation time of conventional PO and the proposed modified PO is comparable. For higher frequency, the modified PO method takes $80 \%$ to $90 \%$ longer time to simulation compared to the conventional PO method. This excess simulation time can be expressed by the fact that for high-frequency simulation, the number of discrete surface points selected is larger. As there are 22 values $\rho_{s}$ selected per wavelength and the wavelength is smaller for high-frequency simulation, the overall number of point increases. The filtering of the surface currents requires this additional simulation time due to large number of points. However, the proposed method is much faster compared to Method of Moments. For each simulation, the Method of Moments requires over 500 seconds of simulation time. 


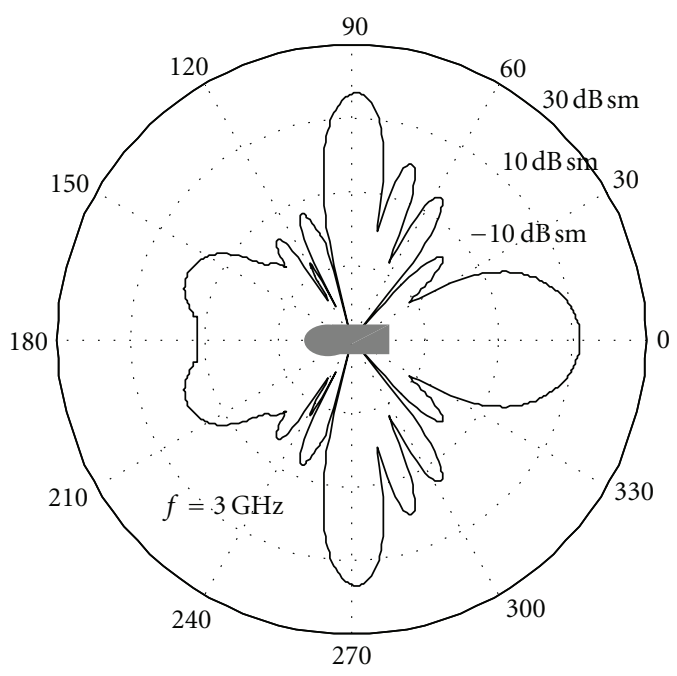

(a)

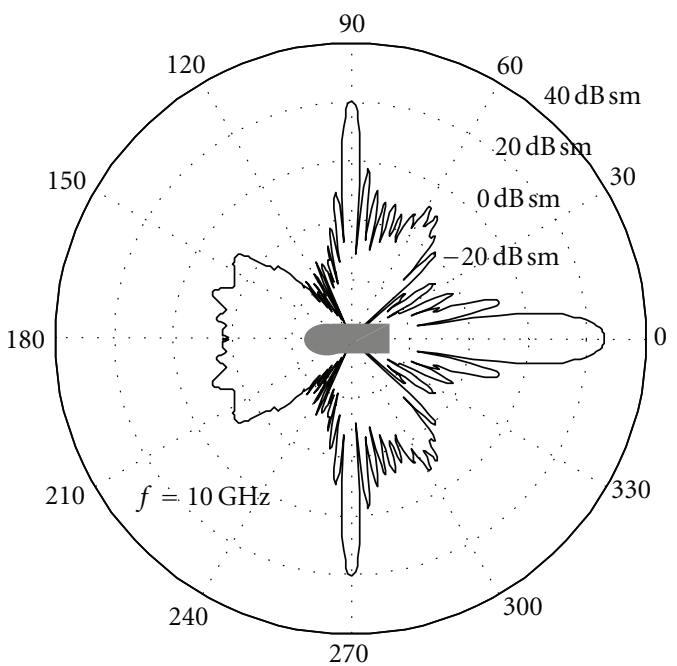

(c)

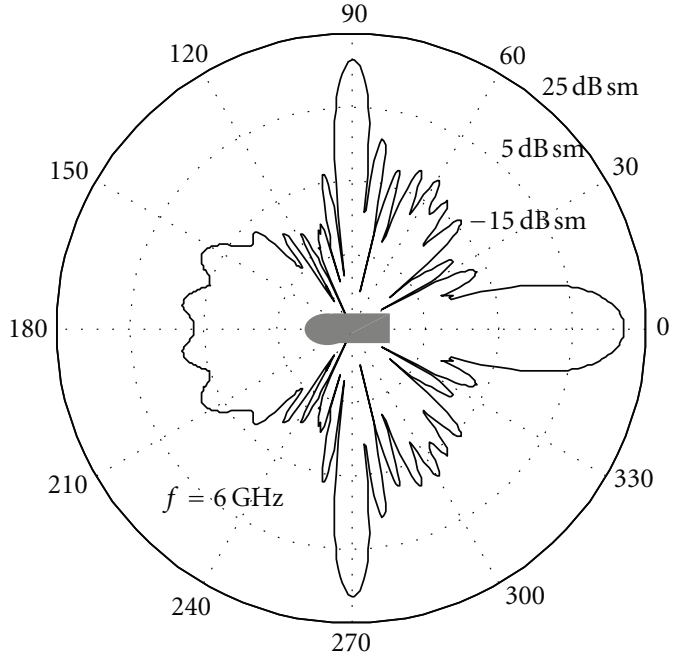

(b)

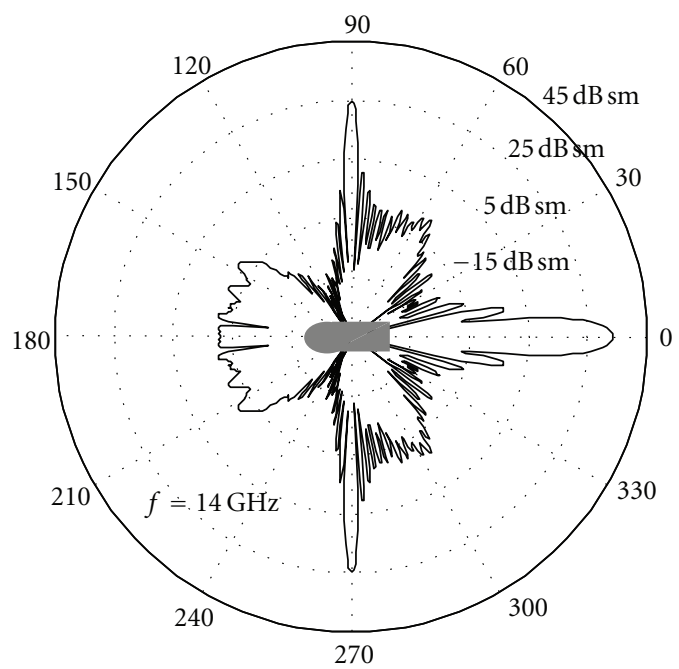

(d)

Figure 11: Monostatic RCS of the projectile at $3 \mathrm{GHz}, 6 \mathrm{GHz}, 10 \mathrm{GHz}$, and $14 \mathrm{GHz}$ as a function of $\theta$.

TABLE 1: Comparison of CPU simulation time.

\begin{tabular}{lcc}
\hline Frequency $(\mathrm{GHz})$ & Simulation time for PO method $(\mathrm{sec})$ & Simulation time for modified PO method $(\mathrm{sec})$ \\
\hline 3 & 16.83 & 26.09 \\
6 & 32.09 & 50.79 \\
10 & 53.19 & 87.01 \\
14 & 74.15 & 144.23 \\
\hline
\end{tabular}

\section{Conclusion}

RCS formulation procedure using PO method is described in detail in this paper. The paper covers geometrical modeling of shell-shaped projectile using differential geometry, formulation of surface current density, evaluation of the scattered field integral in parametric space, and monostatic RCS formulation. A modified PO method is presented which approximates surface current density more accurately. The additional computational steps require Fourier transform and inverse Fourier transform only. These can be easily incorporated in computer code using well-known Fast Fourier Transform (FFT) algorithm. Thus the accuracy is increased without significant increase in computational complexity. The obtained results using the modified PO method are consistent with similar results found in literature. 


\section{References}

[1] E. F. Knott, J. F. Shaeffer, and M. T. Tuley, Rada Cross Section, Scitech Publishing, 2nd edition, 2004.

[2] D. C. Jenn, Radar and Laser Cross Section Engineering, American Institute of Aeronautics and Astronautics (AIAA), 2nd edition, 2005.

[3] C. A. Balanis, Antenna Theory Analysis and Design, John Wiley \& Sons, 3rd edition, 2005.

[4] C. Uluișik, G. Çakir, M. Çakir, and L. Sevgi, "Radar cross section (RCS) modeling and simulation, part 1: a tutorial review of definitions, strategies, and canonical examples," IEEE Antennas and Propagation Magazine, vol. 50, no. 1, pp. 115126, 2008.

[5] G. Çakir, M. Çakir, and L. Sevgi, "Radar cross section (RCS) modeling and simulation, part 2: a novel FDTD-based RCS prediction virtual tool for the resonance regime," IEEE Antennas and Propagation Magazine, vol. 50, no. 2, pp. 81-94, 2008.

[6] C. M. Kuo and C. W. Kuo, "A new scheme for the conformal FDTD method to Calculate the radar cross section of perfect conducting curved objects," IEEE Antennas and Wireless Propagation Letters, vol. 9, pp. 16-19, 2010.

[7] J. Ling, S. X. Gong, X. Wang, B. Lu, and W. T. Wang, "A novel two-dimensional extrapolation technique for fast and accurate radar cross section computation," IEEE Antennas and Wireless Propagation Letters, vol. 9, pp. 244-247, 2010.

[8] J. Ling, S. X. Gong, W. T. Wang, X. Wang, and Y. J. Zhang, "Fast monostatic radar cross section computation using Maehly approximation," IET Science, Measurement and Technology, vol. 5, no. 1, pp. 1-4, 2011.

[9] J. Bao, D. Wang, and E. K. N. Yung, "Electromagnetic scattering from an arbitrarily shaped bi-isotropic body of revolution," IEEE Transactions on Antennas and Propagation, vol. 58, no. 5, pp. 1689-1698, 2010.

[10] J. M. Rius, M. Ferrando, and L. Jofre, "High-frequency RCS of complex radar targets in real-time," IEEE Transactions on Antennas and Propagation, vol. 41, no. 9, pp. 1308-1319, 1993.

[11] D. A. McNamara, C. W. I. Pistorius, and J. A. G. Malherbe, Introduction to The Uniform Geometrical Theory of Diffraction, Artech House, 1990.

[12] L. Angermann, Numerical Simulations-Applications, Examples and Theory, Intech, 2011.

[13] X. J. Chen and X. W. Shi, "An expression for the radar cross section computation of an electrically large perfect conducting cylinder located over a dielectric half-space," Progress in Electromagnetics Research, vol. 77, pp. 267-272, 2007.

[14] S. Blume and G. Kahl, "The physical optics radar cross section of an elliptic cone," IEEE Transactions on Antennas and Propagation, vol. 35, no. 4, pp. 457-460, 1987.

[15] C. Bourlier and P. Pouliguen, "Useful analytical formulae for near-field monostatic radar cross section under the physical optics: far-field criterion," IEEE Transactions on Antennas and Propagation, vol. 57, no. 1, pp. 205-214, 2009.

[16] D. M. Elking, J. M. Roedder, D. D. Car, and S. D. Alspach, "A review of high-frequency radar cross section analysis capabilities at McDonnell Douglas Aerospace," IEEE Antennas and Propagation Magazine, vol. 37, no. 5, pp. 33-43, 1995.

[17] J. Lee, M. Havrilla, M. Hyde, and E. J. Rothwell, "Scattering from a cylindrical resistive sheet using a modified physical optics current," IET Microwaves, Antennas and Propagation, vol. 2, no. 5, pp. 482-491, 2008.
[18] T. Shijo, L. Rodriguez, and M. Ando, "The modified surfacenormal vectors in the physical optics," IEEE Transactions on Antennas and Propagation, vol. 56, no. 12, pp. 3714-3722, 2008.

[19] R. C. Johnson, Chapter 20: Phased Arrays, Antenna Engineering Handbook, McGraw-Hill, 4th edition, 2007, Edited by: J. L. Volakis.

[20] R. Ross, "Radar cross section of rectangular flat plates as a function of aspect angle," IEEE Transactions on Antennas and Propagation, vol. 14, no. 8, pp. 329-335, 1966.

[21] S. D. Weiner and S. L. Borison, "Radar scattering from blunted cone tips," IEEE Transactions on Antennas and Propagation, vol. 14, no. 6, pp. 774-781, 1966.

[22] D. V. Widder, Advanced Calculus, Prentice Hall, 2nd edition, 2004.

[23] T. M. Apostol, Calculus Volume II, John Wiley \& Sons, 2nd edition, 1969.

[24] A. D. Polyanin and A. V. Manzhirov, Handbook of Mathematics for Engineers and Scientists, Chapman \& Hall, 2007.

[25] S. S. Soliman and M. D. Srinath, Continuous and Discrete Signals and Systems, Prentice Hall, 2007.

[26] M. Andreasen, "Scattering from bodies of revolution," IEEE Transactions on Antennas and Propagation, vol. 13, no. 2, pp. 303-310, 1965. 

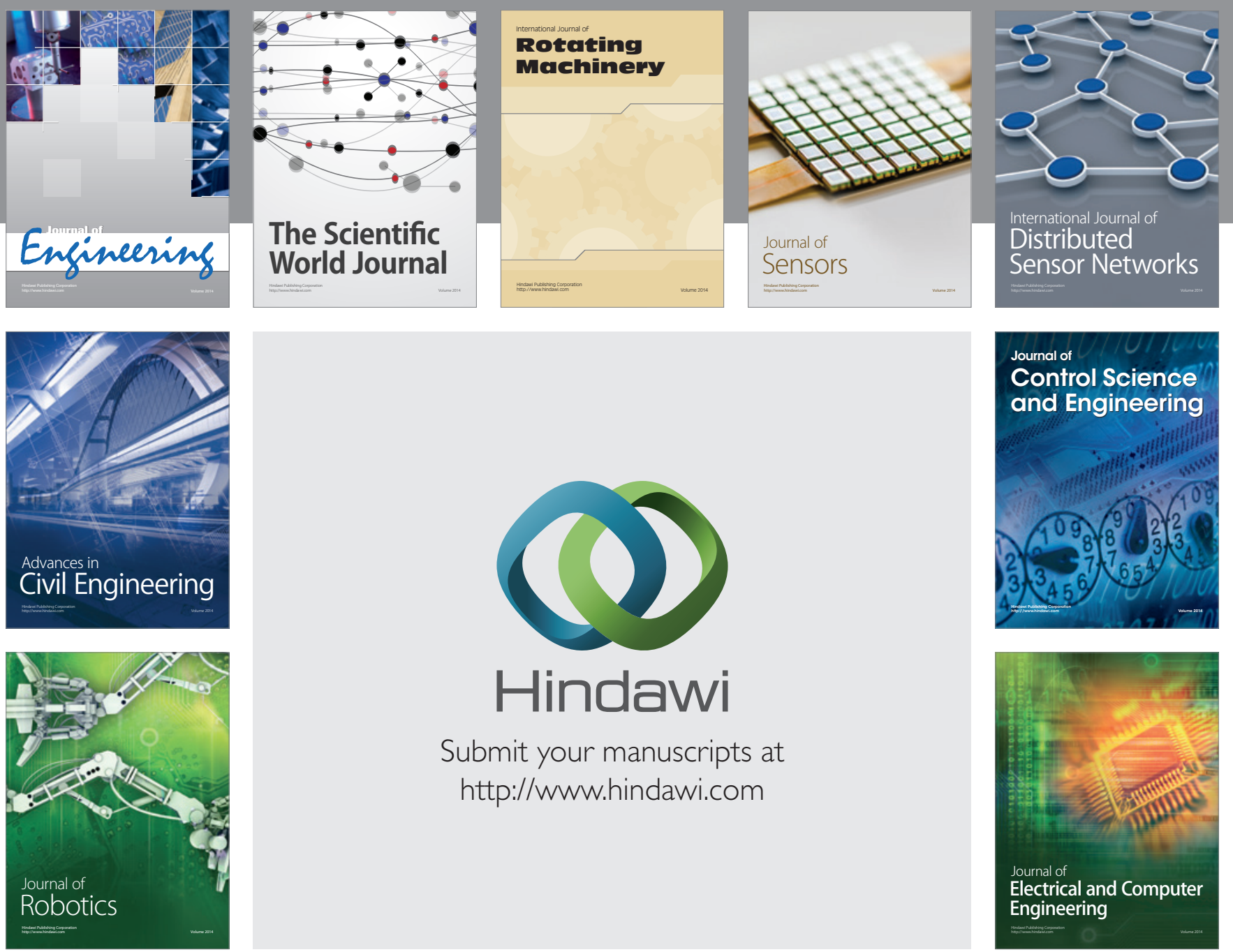

Submit your manuscripts at

http://www.hindawi.com
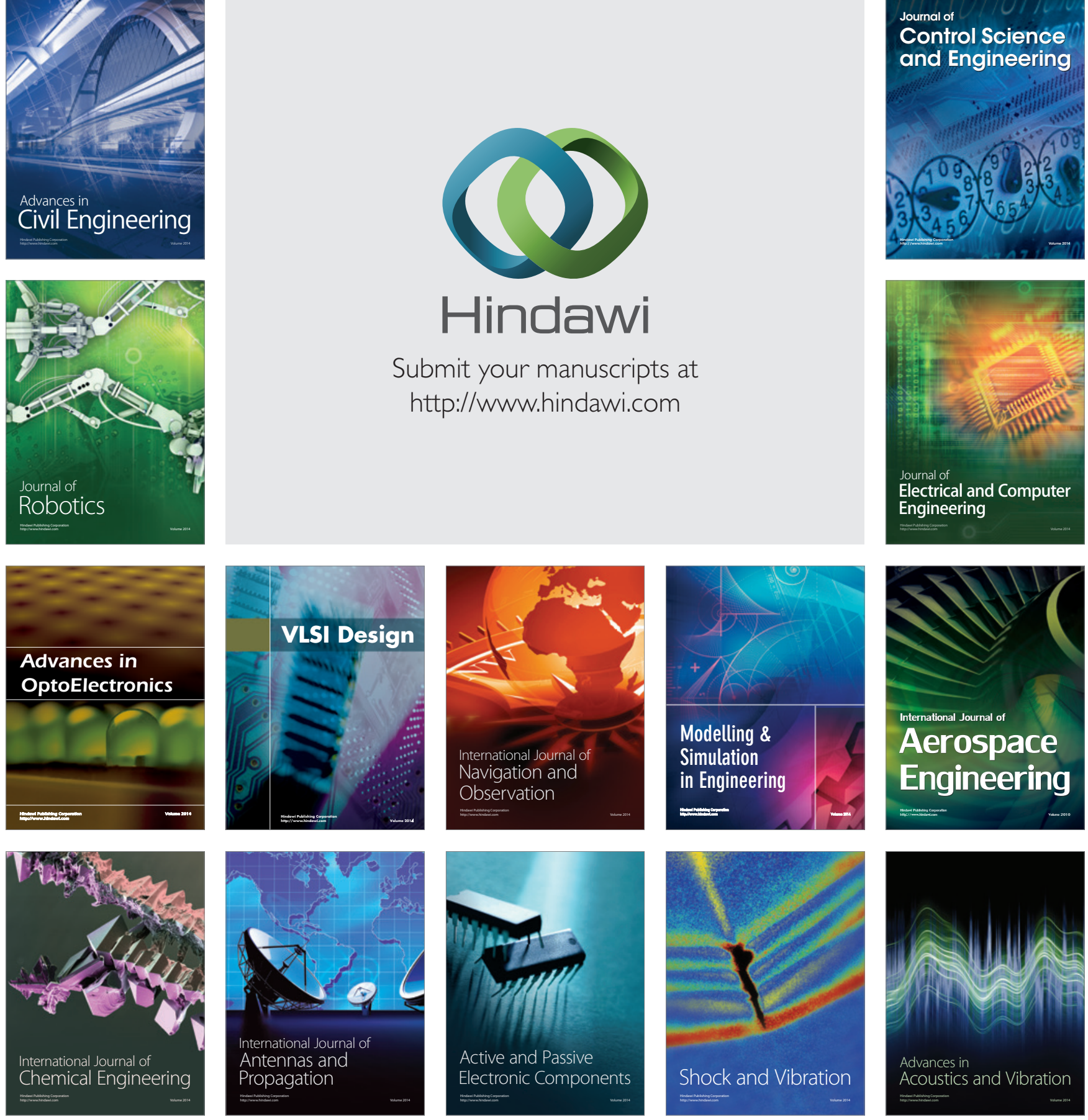